Bull. Austral. Math. Soc.

VOL. $74(2006) \quad[289-300]$

\title{
LINEAR RELATIONS ON HEREDITARILY INDECOMPOSABLE NORMED SPACES
}

\author{
TEResa Álvarez
}

\begin{abstract}
We introduce the notion of hereditarily indecomposable normed space and we prove that this class of normed spaces may be characterised by means of $F_{+}$and strictly singular linear relations. We also show that if $X$ is a complex hereditarily indecomposable normed space then every partially continuous linear relation in $X$ with dense domain can be written as $\lambda I+S$, where $\lambda \in \mathbb{C}$ and $S$ is a strictly singular linear relation.
\end{abstract}

\section{INTRODUCTION}

It has been an open question for some time whether every infinite dimensional Banach space $E$ is decomposable; that is, whether we can write $E=M \oplus N$, with $M$ and $N$ infinite dimensional closed subspaces. In [9] Gowers and Maurey constructed the first example of a hereditarily indecomposable (HI for short) Banach space, that is, a space with no decomposable closed subspaces.

A result, essentially due to Weiss ([15], contains the hard implications) characterises the HI Banach spaces as those spaces $E$ such that for every Banach space $F$ any bounded operator from $E$ into $F$ is upper semiFredholm or strictly singular. Note that, at the time Weiss proved this result, the existence of hereditarily indecomposable Banach spaces was an open problem. Furthermore, Gowers an Maurey [9] showed that for a complex HI Banach space $E$ the bounded operators in $E, B[E]$, have a very simple structure: $B[E]=\mathbb{C} I+S S[E]$ where $S S[E]$ is the class of all bounded strictly singular operators in $E$.

The aim of this paper is to find conditions under which results of the type mentionated above will still be true in the much more general setting of multivalued linear operators between normed spaces.

In Section 2 we define the notion of $H I$ normed space which generalises the classical definition of Gowers and Maurey [9]. We also introduce and present some properties of essential spectrums of linear relations. These properties together with some results of perturbation for $F_{+}$and $F_{-}$linear relations will be applied in Section 3 to obtain the

Received 9th May, 2006

Copyright Clearance Centre, Inc. Serial-fee code: 0004-9727/06 \$A2.00+0.00. 
main results (Theorems 17 and 18 below) which generalise similar results of Weiss [15] and Gowers and Maurey [9] for bounded operators between Banach spaces.

Notations. We adhered to the notation and terminology of the book [4]: $X, Y$ are infinite dimensional normed spaces over $\mathbb{K}=\mathbb{R}$ or $\mathbb{C}, X^{\prime}$ the dual space of $X$. If $M \subset X$ and $N \subset X^{\prime}$, then

$$
M^{\perp}:=\left\{x^{\prime} \in X^{\prime}: x^{\prime}(x)=0 \text { for all } x \in M\right\}
$$

and

$$
N^{\top}:=\left\{x \in X: x^{\prime}(x)=0 \text { for all } x^{\prime} \in N\right\} .
$$

Let $I(X), S(X), C(X)$ and $P(X)$ denote the infinite dimensional, infinite dimensional closed, finite codimensional and finite codimensional closed subspaces of $X$, respectively.

A linear relation or multivalued linear operator $T: X \rightarrow Y$ is a mapping from a subspace $D(T) \subseteq X$, called the domain of $T$, into the collection of nonempty subsets of $Y$ such that $T\left(\alpha x_{1}+\beta x_{2}\right)=\alpha T x_{1}+\beta T x_{2}$ for all nonzero $\alpha, \beta$ scalars and $x_{1}, x_{2} \in D(T)$. The class of such linear relations $T$ is denoted by $L R(X, Y)$. If $T$ maps the points of its domain to singletons, then $T$ is said to be a single valued or simply an operator.

The graph $G(T)$ of $T \in L R(X, Y)$ is defined by

$$
G(T):=\{(x, y) \in X \times Y: x \in D(T), y \in T x\}
$$

which is a subspace of $X \times Y$. Let $M$ be a subspace of $D(T)$. Then the restriction $\left.T\right|_{M}$ is defined in terms of its graph by

$$
G\left(\left.T\right|_{M}\right):=\{(m, y) \in M \times Y: y \in T m\} .
$$

For any subspace $M$ of $X$ such that $M \cap D(T) \neq \emptyset$, we write $\left.T\right|_{M}=\left.T\right|_{M \cap D(T)}$. We note that $\left.T\right|_{M} \in L R(X, Y)$ but $T J_{M} \in L R(M, Y)$ where $J_{M}$ denotes the natural injection map of $M$ into $X$. The inverse of $T$ is the linear relation $T^{-1}$ defined by

$$
G\left(T^{-1}\right)=\{(y, x) \in Y \times X:(x, y) \in G(T)\} .
$$

If $T^{-1}$ is single valued, then $T$ is called injective, that is, $T$ is injective if and only if its null space $N(T):=T^{-1}(0)=\{0\}$ and $T$ is said to be surjective if its range

$$
R(T):=T(D(T))=Y \text {. }
$$

The conjugate $T^{\prime}$ of $T$ is defined by $G\left(T^{\prime}\right):=G\left(-T^{-1}\right)^{\perp}$, where

$$
\left\langle(y, x)\left(y^{\prime}, x^{\prime}\right)\right\rangle:=\left\langle x, x^{\prime}\right\rangle+\left\langle y, y^{\prime}\right\rangle .
$$

This means that $\left(y^{\prime}, x^{\prime}\right) \in G\left(T^{\prime}\right)$ if and only if $y^{\prime}(y)-x^{\prime}(x)=0$ for all $(x, y) \in G(T)$. 
For $T \in L R(X, Y), \widetilde{T}$ denotes the linear relation defined by $G(\widetilde{T}):=\widetilde{G(T)}$ $C \tilde{X} \times \tilde{Y}$ where $\tilde{X}$ denotes the completion of $X$. We define $\alpha(T):=\operatorname{dim} N(T)$; $\beta(T):=\operatorname{dim} Y / R(T) ; \bar{\beta}(T):=\operatorname{dim} Y / \overline{R(T)} ; k(T):=\alpha(T)-\beta(T)$ (if either $\alpha(T)$ or $\beta(T)$ are finite) and $\bar{k}(T):=\alpha(\widetilde{T})-\alpha\left(T^{\prime}\right)$ (if either $\alpha(\widetilde{T})$ or $\alpha\left(T^{\prime}\right)$ are finite).

For a given closed subspace $Z$ of $X$ let $Q_{Z}^{X}$ or simply $Q_{Z}$ denote the natural quotient map from $X$ onto $X / Z$. We shall denote $Q_{\overline{T(0)}}$ by $Q_{T}$. Clearly $Q_{T} T$ is single valued. For $x \in D(T),\|T x\|:=\left\|Q_{T} T x\right\|$ and the norm of $T$ is defined by $\|T\|:=\left\|Q_{T} T\right\|$.

A linear relation $T \in L R(X, Y)$ is said to be

1. closed if its graph is closed,

2. continuous if $\|T\|<\infty$,

3. open if $T^{-1}$ is continuous (equivalently $\gamma(T)>0$ where $\gamma(T)$ is the minimum modulus of $T$ defined by $\gamma(T):=\sup \{\lambda \geqslant 0: \lambda \operatorname{dis}(x, N(T))$ $\leqslant\|T x\|$ for all $x \in D(T)\}$ ),

4. partially continuous if there exists $M \in C(X)$ such that $\left.T\right|_{M}$ is continuous,

5. strictly singular if there is no $M \in I(D(T))$ for which $\left.T\right|_{M}$ is injective and open,

6. $F_{+}$if there exists $M \in C(X)$ such that $\left.T\right|_{M}$ is injective and open,

7. upper semiFredholm if $R(T)$ is closed and $\operatorname{dim} N(T)<\infty$,

8. $F_{-}$if $T^{\prime}$ is $F_{+}$, lower semiFredholm if $R(T)$ is finite codimensional closed,

9. lower semiFredholm if $R(T)$ is finite codiemnsional closed.

The classes of partially continuous, strictly singular, $F_{+}$upper semiFredholm, $F_{-}$and lower semiFredholm linear relations will be denoted by $P B(X, Y), S S(X, Y), F_{+}(X, Y)$, $\phi_{+}(X, Y), F_{-}(X, Y)$ and $\phi_{-}(X, Y)$, respectively.

Square brackets will be used to indicate that only everywhere defined linear relations are considered; for example $B R[X, Y]$ denotes the class of everywhere defined continuous (bounded, for short) linear relations in $L R(X, Y)$ and we also write

$$
\begin{aligned}
B[X, Y] & :=\{T \in B R[X, Y]: T \text { single valued }\} \text { and } \\
P B_{D}(X, Y) & :=\{T \in P B(X, Y): \overline{D(T)}=X\} .
\end{aligned}
$$

An underlying motivation for the introduction of multivalued linear operators into Functional Analysis by J. von Neumann [13] was to aid the investigation of differential equations governed by non densely defined operators. The conjugate of such operators are linear relations. Linear relations are more convenient because one can define the inverse, the closure and the completion of a linear relation. Interesting works on multivalued linear operators include the treatise of partial differential relations by Gromov [10], the application of multivalued methods to solution of differential equations by Favini and Yagi [6], the development of fixed point theory for linear relations to the existence of mild solutions of quasi-linear differential inclusions of evolution and also to many problems of 
fuzzy theory (see, for example, $[1,8,12,14]$ ); and several papers on linear relations type semiFredholm and other classes related to them $[2,3,4]$, among others.

\section{BASIC DEFINITIONS AND ESSENTIAL SPECTRUMS}

The following lemmata are elementary but they help to read Definition 3.

Lemma 1. Let $M$ be a closed subspace of $X$. We have:

(i) $(X / M)^{\prime}$ is isometrically isomorphic to $M^{\perp}$.

(ii) Let $N$ be a subspace of $X$ such that $M \subset N$. Then $N$ is closed if and only if $N / M$ is closed in $X / M$.

Proof:

(i) See $[7$, I.6.4].

(ii) The proof proceeds by direct computation.

LEMMA 2. A Banach space $E$ is hereditarily indecomposable if and only if there are no infinite dimensional closed subspaces $M$ and $N$ of $E$ such that $Q_{M} J_{N}$ is injective and open.

Proof: We first note that by the Open Mapping Theorem we have the following property:

(1) If $T: X \rightarrow Y$ is a bounded single valued where $X$ and $Y$ are Banach spaces, then $T$ is open if and only if $R(T)$ is closed.

Let $M, N \in S(E)$. Then the null space of $Q_{M} J_{N}$ is $M \cap N$ and its range is $M+N / M$ and hence the result follows from Lemma 1 and property (1).

This Lemma suggests the following notion.

Definition 3: A normed space $X$ is said to be hereditarily indecomposable (HI for short) if there are no $M, N \in S(X)$ such that $Q_{M} J_{N}$ is injective and open.

Proposition 4. Let $K \in L R(X)$ be a precompact single valued and 0 $<\varepsilon<1 / 2$ such that $\|K\|<\varepsilon$. Then $I+K$ is injective, open with dense range and $(I+K)^{-1}=I+K_{1}$ with $K_{1}$ precompact and $\left\|K_{1}\right\|<2 \varepsilon$.

Proof: Since $\|K\|<\gamma(I)$, we have that $I+K$ is injective, open with dense range by [4, III.7.7]. Moreover, from

$$
I=(I+K)^{-1}(I+K)=(I+K)^{-1}+(I+K)^{-1} K,
$$

it follows that

$$
(I+K)^{-1}=I-(I+K)^{-1} K:=I+K_{1} \text { with } K_{1}:=-(I+K)^{-1} K
$$

precompact and

$$
\left\|(I+K)^{-1}\right\|<1+\varepsilon\left\|(I+K)^{-1}\right\| .
$$


Hence

$$
\left\|(I+K)^{-1}\right\|<1 /(1-\varepsilon)<2
$$

and consequently $\left\|K_{1}\right\|<2 \varepsilon$, as desired.

Definition 5: ([4, IV.1.1]) For a given linear relation $T \in L R(X, Y)$ we define the quantities $\Gamma(T)$ and $\Delta(T)$ as follows: If $\operatorname{dim} D(T)<\infty$, then $\Gamma(T):=\Delta(T):=0$ and if $\operatorname{dim} D(T)=\infty$, then

$$
\begin{aligned}
\Gamma(T) & :=\inf \left\{\left\|\left.T\right|_{M}\right\|: M \in I(D(T))\right\} \text { and } \\
\Delta(T) & :=\sup \left\{\Gamma\left(\left.T\right|_{M}\right): M \in I(D(T))\right\} .
\end{aligned}
$$

Proposition 6. [4, V.2.4 and V.2.6] Let $T \in L R(X, Y)$. Then

(i) If $D(T)$ is infinite dimensional, $T \in F_{+}$if and only if $\Gamma(T)>0$.

(ii) $T \in S S(X, Y)$ if and only if $\Delta(T)=0$.

The notions of essential spectrums given in Edmunds and Evans [5] for single valued can be extended to linear relations as follows:

Definition 7: Let $T \in L R(X)$ where $X$ is a normed space over the complex field C. We define the essential resolvents, $\rho_{e+}(T)$ and $\rho_{e}(T)$ of $T$ by

$$
\begin{aligned}
\rho_{e+}(T) & :=\left\{\lambda \in \mathbb{C}: \lambda-T \in F_{+}\right\}, \\
\rho_{e}(T) & :=\left\{\lambda \in \mathbb{C}: \lambda-T \in F_{+} \cap F_{-} \text {and } \bar{k}(\lambda-T)=0\right\} .
\end{aligned}
$$

The essential spectrums $\sigma_{e+}(T)$ and $\sigma_{e}(T)$ are the respective complements of the essential resolvents:

$$
\sigma_{e+}(T):=\mathbb{C} \backslash \rho_{e+}(T) \text { and } \sigma_{e}(T):=\mathbb{C} \backslash \rho_{e}(T) .
$$

We recall that the resolvent set of $T$ is defined by Cross [4, VI.1.1] as

$$
\rho(T):=\{\lambda \in \mathbb{C}: \lambda-T \text { is injective, open and has dense range }\}
$$

and the spectrum of $T$ is the set $\sigma(T):=\mathbb{C} \backslash \rho(T)$.

Since every open and injective (respectively, open with dense range) multivalued linear operator is $\boldsymbol{F}_{+}[\mathbf{4}, \mathrm{V} .5 .1]$ (respectively, $\boldsymbol{F}_{-}[\mathbf{4}, \mathrm{V} .5 .2]$ ) we have the inclusions $\sigma_{e+}$ $(T) \subset \sigma_{e}(T) \subset \sigma(T)$ for $T \in L R(X)$ where $X$ is a complex normed space.

Lemma 8. Let $T \in L R(X, Y)$ be closed and let $S \in L R(X, Y)$ be continuous such that $\overline{D(T)} \subset D(S)$ and $S(0) \subset T(0)$. Then $T+S$ is closed.

Proof: Assume first that $T$ and $S$ are single valued. Let $\left(x_{n}\right)$ be a sequence in $D(T+S)=D(T) \cap D(S)=D(T)$ such that $x_{n} \rightarrow x$ and $(T+S) x_{n} \rightarrow y$ for some $x \in X$ and $y \in Y$. Then $x \in \overline{D(T)} \subset D(S)$ and $S x_{n} \rightarrow S x$ since $S$ is continuous. Thus 
$T x_{n} \rightarrow S x-y$ and as $T$ is closed, we have that $x \in D(T)$ and $T x=S x-y$, that is, $T+S$ is closed. For the general case, we observe that $Q_{T} T$ is a closed operator and $T(0)$ is closed ([4, II.5.3]), $(T+S)(0)=T(0)$ because $S(0) \subset T(0)$ and $Q_{T} S=Q_{T(0) / \overline{S(0)}} Q_{S} S$ by $\left[4\right.$, IV.5.2]. Hence, from what has been proved for the single valued case, $Q_{T+S}(T+S)$ $=Q_{T} T+Q_{T} S$ is closed and since $(T+S)(0)$ is closed, applying again [4, II.5.3] we obtain that $T+S$ is closed, as desired.

LEMMA 9. Let $T, S \in L R(X, Y)$. Then

(i) If $\overline{D(T)} \subset D(S)$ is $S^{\prime}(0) \subset T^{\prime}(0)$.

(ii) If $S$ is continuous and $S(0) \subset \overline{T(0)}$, then $\overline{D\left(T^{\prime}\right)} \subset D\left(S^{\prime}\right)$.

Proof:

(i) Since $S^{\prime}(0)=D(S)^{\perp}([4$, III.1.4]) and $\overline{D(T)} \subset D(S)$ it follows trivially the assertion (i).

(ii) Let $S$ be continuous. Then $\left\|S^{\prime}\right\|=\|S\|\left(\left[4\right.\right.$, III.1.13]) and $D\left(S^{\prime}\right)$ $=S(0)^{\perp}\left(\left[4\right.\right.$, III.1.4]). If moreover $S(0) \subset \overline{T(0)}$ we obtain that $\overline{D\left(T^{\prime}\right)}$ $\subset\left(D\left(T^{\prime}\right)\right)^{\top \perp}=\bar{T}(0)^{\perp}\left([4\right.$, III.1.4] $) \subset \overline{T(0)} \bar{\perp}^{\perp}\left(\left[4\right.\right.$, II.5.19]) $\subset S(0)^{\perp}$ $=D\left(S^{\prime}\right)$.

Proposition 10. Let $T \in F_{+}(X, Y)$ (respectively, $T \in F_{-}(X, Y)$ and let $S$ $\in L R(X, Y)$ such that $\overline{D(T)} \subset D(S), S(0) \subset \overline{T(0)}$ and $\|S\|<\gamma(\widetilde{T})$. Then $T+S$ $\in F_{+}(X, Y)$ (respectively, $T+S \in F_{-}(X, Y)$ ) and $\bar{k}(T+S)=\bar{k}(T)$.

Proof: By [4, V.7.6 and V.1.9], $\widetilde{T} \in \phi_{+}(\tilde{X}, \tilde{Y})$ (repectively, $\tilde{T} \in \phi_{-}(\tilde{X}, \tilde{Y})$ ), and hence, by the Open Mapping Theorem [4, III.4.2], $\gamma(\widetilde{T})>0$. Furthermore, we note that $\|\tilde{S}\| \leqslant\|S\|$. Thus we may assume that $X$ and $Y$ are Banach spaces, that $T$ is closed, and show the stability holds for $k(T)$. Since $S$ is continuous, $\overline{D(T)} \subset D(S)$ and $S(0) \subset \overline{T(0)}$, $T+S$ is closed by Lemma 8 .

CASE I. $T \in F_{+}$. The proof of this case is along the lines of the proof of the homologous result proved in $[4, V .15 .6]$ for the case when $S$ is single valued, with the appropriate modifications.

Since $T_{+} \in \phi_{+}$, by $\left[4\right.$, V.3.2] is $T+S \in F_{+}$, and since $T+S$ is closed, $T+S \in \phi_{+}$. Then there is $M \in P(D(T))$ such that $\left.T\right|_{M}$ is injective and open. Since $M$ is closed, $\left.T\right|_{M}$ is closed, and it follows from the Open Mapping Theorem that $R\left(\left.T\right|_{M}\right)$ is closed, that is, $\left.T\right|_{M} \in \phi_{+}$. We first deduce the conclusion for the case $\|S\|<\gamma\left(\left.T\right|_{M}\right)$. Applying $\left[4\right.$, V.3.2], $\left.(T+S)\right|_{M} \in F_{+}$and by Lemma $8, T+S$ is closed and hence $\left.(T+S)\right|_{M} \in \phi_{+}$. Thus by $[4$, III.7.6],

$$
\beta\left(\left.(T+S)\right|_{M}\right)=\bar{\beta}\left(\left.(T+S)\right|_{M}\right)=\bar{\beta}\left(\left.T\right|_{M}\right)=\beta\left(\left.T\right|_{M}\right)
$$

and it now follows from the finite dimensional extension lemma [4, V.15.5] that

$$
k(T+S)=k\left(\left.(T+S)\right|_{M}\right)+\alpha(T)=k\left(\left.T\right|_{M}\right)+\alpha(T)=k(T)
$$


provided $\|S\|<\gamma\left(\left.T\right|_{M}\right)$.

If now $\|S\|<\gamma(T)$, let $I$ denote the closed interval $[0,1]$ with the usual topology and let $Z:=\mathbb{Z} \cup\{-\infty,+\infty\}$ with the discrete topology, and define

$$
\theta: \lambda \in I \rightarrow \theta(\lambda):=k(T+\lambda S) \in Z .
$$

It follows from the above that

$$
\theta(\lambda)=k\left(T+\lambda_{0} S+\left(\lambda-\lambda_{0}\right) S\right)=k\left(T+\lambda_{0} S\right)=\theta\left(\lambda_{o}\right)
$$

provided $\lambda_{o}$ is sufficiently close to $\lambda$. Hence, $\theta$ is continuous and $\theta(I)$ is a connected set and therefore consists of just one point. Hence

$$
k(T)=\theta(0)=\theta(1)=k(T+S)
$$

as required.

CASE II. $T \in F_{-}$. Then $T^{\prime} \in \phi_{+}$and $R\left(T^{\prime}\right)$ is closed. Since $S$ is continuous, $D(T)$ $\subset D(S)$, we have that $(T+S)^{\prime}=T^{\prime}+S^{\prime}\left(\left[4\right.\right.$, III.1.5]). Furthermore, $\left\|S^{\prime}\right\| \leqslant\|S\|$ $<\gamma(T)=\gamma\left(T^{\prime}\right)\left(\left[4\right.\right.$, III.4.6]). Thus, by Case I, $R\left(T^{\prime}+S^{\prime}\right)$ is closed and

$$
k(T)=-k\left(T^{\prime}\right)=-k\left(T^{\prime}+S^{\prime}\right)=k\left((T+S)^{\prime}\right)-k(T+S),
$$

as desired.

Proposition 11. Let $X$ be a complex normed space and let $T \in L R(X)$. Then $\sigma(T), \sigma_{e+}(T)$ and $\sigma_{e}(T)$ are closed.

Proof: $\rho(T)$ open. See [4, VI.1.3].

$\rho_{e+}(T)$ open. Indeed, clearly $\rho_{e+}(T)=\mathbb{C}$ if $\operatorname{dim} D(T)<\infty$. Hence assume that $\operatorname{dim} D(T)=\infty$ and let $\lambda \in \rho_{e+}(T)$. Then $\lambda-T \in F_{+}$equivalently $\Gamma(\lambda-T)>0$ (Proposition 6). If $\delta \in \mathbb{C}$ such that $|\delta-\lambda|<\Gamma(\lambda-T)$, then

$$
\Delta((\delta-\lambda) I) \leqslant\|(\delta-\lambda) I\| \leqslant \Gamma(\lambda-T)
$$

and so it follows from [4, V.3.2] that $\delta-T \in F_{+}$, that is, $\rho_{e+}(T)$ is open and hence $\sigma_{e+}(T)$ is closed, as desired.

$\rho_{e}(T)$ open. Let $\lambda \in \rho_{e}(T)$, that is, $\lambda-T \in F_{+} \cap F_{-}$(so that, $\gamma(\lambda-\widetilde{T})>0$ ) and $\bar{k}(\lambda-T)=0$. Then, if $\delta \in \mathbb{C}$ such that $|\delta-\lambda|<\gamma(\lambda-\tilde{T})$ we obtain from Proposition 10 that $\delta \in \rho_{e}(T)$. Therefore $\rho_{e}(T)$ is open and so $\sigma_{e}(T)$ is closed, as required.

An example of a densely defined single valued $T$ with empty spectrum has been constructed by Cross $\left[4\right.$, V.2.7] and since $\sigma_{e+}(T) \subset \sigma_{e}(T) \subset \sigma(T)$ we conclude that this operator of Cross has empty essential spectrums. This shortcoming falls away in the presence of partially continuous, as the following result shows. 
Proposition 12. Let $X$ be a complex normed space, and let $T$ be partially continuous and densely defined linear relation in $L R(X)$. Then $\sigma(T), \sigma_{e}(T)$ and $\sigma_{e+}(T)$ are non empty.

Proof: $\sigma(T) \neq \emptyset$. Indeed, $T$ partially continuous $\Rightarrow T^{\prime}$ continuous $[4$, V.9.6] and since $T^{\prime}(0)=D(T)^{\perp}$ [4, III.1.4] we deduce that $T^{\prime}$ is continuous and single valued equivalently $\widetilde{T}$ is bounded $[4$, VIII.1.5] and thus by virtue of $[4$, VI3.3], $\sigma(\widetilde{T}) \neq \emptyset$. But as $\sigma(\widetilde{T})=\sigma(T)[4$, VI.1.1], $\sigma(T) \neq \emptyset$, as desired.

$\sigma_{e}(T) \neq \emptyset$. We first prove the following properties:

(2) $T \in L R(X) \Rightarrow \sigma_{e}(T)=\sigma_{e}\left(T^{\prime}\right)$.

This assertion follows from the definitions upon noting that $T \in F_{-} \Leftrightarrow T^{\prime} \in F_{+}$, $T \in F_{+} \Leftrightarrow T^{\prime} \in F_{-}\left(\left[4, \mathrm{~V} .1 .1\right.\right.$ and V.7.5]) and that if $R\left(T^{\prime}\right)$ is closed then $\bar{k}(T)=-k\left(T^{\prime}\right)$ whenever either quantity exists $([4, \mathrm{~V} .15 .3])$.

(3) T partially continuous and densely defined $\Rightarrow T^{\prime \prime}$ bounded and single valued.

Arguing as in the previous case, we have that $T^{\prime}$ is single valued and continuous and hence $T^{\prime \prime}$ is a bounded single valued.

Now, it is well known that given a complex Banach space $E$ and a bounded operator $T \in B[E], \sigma_{e}(T)$ coincides with the spectrum of the image of $T$ in the Calkin algebra $B[E] / K[E]([11,53])$, where $K[E]$ denotes the class of all bounded compact operators on $E$. Combining this observation with the properties $\sigma(T) \neq \emptyset$, (2) and (3) we deduce that $\sigma_{e}(T) \neq \emptyset$.

$\sigma_{e+}(T) \neq \emptyset$. Since $\sigma(T)$ is closed and non empty to prove that $\sigma_{e+}(T)$ is non empty it is sufficient to verify that the boundary of $\sigma_{e}(T), \sigma_{e}(T)^{b}$, is contained in $\sigma_{e+}(T)$. Let

$$
\lambda \in \sigma_{e}(T)^{b}=\overline{\sigma_{e}(T)} \cap \overline{\mathbb{C} \backslash \sigma_{e}(T)}=\sigma_{e}(T) \cap \mathbb{C} \backslash \sigma_{e}(T)^{\circ} .
$$

Assume $\lambda \notin \sigma_{e+}(T)$. Then $\lambda-T \in F_{+}$equivalently $\lambda-\widetilde{T} \in \phi_{+}$and hence $\gamma(\lambda-\widetilde{T})>0$. Now, if $|\delta-\lambda|<\gamma(\lambda-\widetilde{T})$ it follows from Proposition 10 that $\delta-T \in F_{+}$and $\bar{k}(\delta-T)$ $=\bar{k}(\lambda-T)$. But $\bar{k}(\lambda-T) \neq 0$ since if $\bar{k}(\lambda-T)=0$ then $\beta(\lambda-\widetilde{T})=\alpha(\lambda-\widetilde{T})<\infty$ which implies that $\lambda-T \in F_{+} \cap F_{-}\left(\left[4\right.\right.$, VII.1.2]) and so $\lambda \in \rho_{e}(T)$ in contradiction with $\lambda \in \sigma_{e}(T)$. In consequence $\bar{k}(\delta-T) \neq 0$, and hence $\lambda \in \sigma_{e}(T)^{\circ}$ which contradicts that $\lambda \in \sigma_{e}(T)^{b}$.

\section{LINEAR RELATIONS ON HI NORMED SPACES}

We recall the following result, essentially due to Weiss [15] which characterises the $H I$ Banach spaces in terms of bounded upper semiFredholm operators.

Proposition 13. A Banach space $E$ is $H I$ if and only if for every Banach space $F, B[E, F]=\phi_{+}[E, F] \cup S S[E, F]$.

We extend (Theorem 17 below) this result to partially continuous linear relations in normed spaces. For this end, we first prove some auxiliary results. 
Proposition 14. If $X$ is a $H I$ normed space, then for every normed space $Y$ and every bounded single valued $T \in B[X, Y]$, is $\Delta(T)=\Gamma(T)$.

Proof: Clearly $\Gamma(T) \leqslant \Delta(T)<\infty$ (as $T$ is continuous). Assume that $X$ is $H I$ and let $M, N \in S(X)$, so that $Q_{M} J_{N}$ is not injective and open. Then, by this property and the Hahn-Banach theorem, the sequences $\left(x_{n}\right)$ in $N,\left(y_{n}\right)$ in $M$ and $\left(x_{n}^{\prime}\right)$ in $X^{\prime}$, can be constructed as follows:

Let $0<\varepsilon<1 / 2$ and select $x_{1} \in N, y_{1} \in M$ and $x_{1}^{\prime} \in X^{\prime}$ such that $1=\left\|x_{1}^{\prime}\right\|$ $=\left\|x_{1}\right\|=x_{1}^{\prime}\left(x_{1}\right)$ and $\left\|y_{1}-x_{1}\right\|<\varepsilon / 2$. Since

$$
N\left(x_{1}^{\prime}\right) \in P(X), N_{1}:=N \cap N\left(x_{1}^{\prime}\right) \in P(N)
$$

and thus there exist $x_{2} \in N_{1}, y_{2} \in M$ and $x_{2}^{\prime} \in X^{\prime}$ such that $1=\left\|x_{2}^{\prime}\right\|=\left\|x_{2}\right\|=x_{2}^{\prime}\left(x_{2}\right)$ and $\left\|y_{2}-x_{2}\right\|<\varepsilon / 2^{2}$. Continuing in this way, we obtain

$$
1=\left\|x_{n}^{\prime}\right\|=\left\|x_{n}\right\|=x_{n}^{\prime}\left(x_{n}\right),\left\|y_{n}-x_{n}\right\|<\varepsilon / 2^{n}
$$

with

$$
y_{n} \in M, x_{n} \in N_{n-1}:=N \cap\left(\bigcap_{i=1}^{n-1} N\left(x_{i}^{\prime}\right)\right), \quad n \in \mathbb{N} .
$$

Clearly the set $\left\{x_{n}: n \in \mathbb{N}\right\}$ is linearly independent. Let $P_{n}$ denote the projection defined on $N$ with range $N_{n}$ and null space $s p\left\{x_{1}, x_{2}, \ldots, x_{n}\right\}$. Then $P_{n}$ is bounded and we can choose $f_{n} \in X^{\prime}$ such that $f_{n}$ is an extension of $x_{n}^{\prime} \circ P_{n}$ with the same norm. Hence

$$
f_{n}\left(x_{m}\right)=\delta_{n m},\left\|f_{n}\right\|\left\|y_{n}-x_{n}\right\|<\varepsilon / 2^{n}
$$

and thus we can consider the nuclear operator

$$
K: x \in X \rightarrow K(x):=\sum_{n=1}^{\infty} f_{n}(x)\left(y_{n}-x_{n}\right) \in X .
$$

It is clear that $K$ is a bounded precompact operator with $\|K\|<\varepsilon<1 / 2$ and $(I+K) x_{n}=y_{n}, n \in \mathbb{N}$. By proposition $4, I+K$ is injective, open with dense range and $\left\|(I+K)^{-1}\right\|<1+2 \varepsilon$. Thus $I+K$ induces an injective open map $\psi$ from $A:=\operatorname{sp}\left\{x_{n}\right\}$ onto $B:=s p\left\{y_{n}\right\}$ such that $(I+K) J_{A}=J_{B} \psi$. Hence

$$
\begin{aligned}
\left\|\left.T\right|_{B}\right\|=\left\|\left.T\right|_{B} \psi \psi^{-1}\right\| & \leqslant\left\|\left.T(I+K)\right|_{A}\right\|\left\|\psi^{-1}\right\| \\
& \leqslant\left(\left\|\left.T\right|_{A}\right\|+\varepsilon\|T\|\right)\left\|\psi^{-1}\right\| \\
& <(1+2 \varepsilon)\left(\left\|\left.T\right|_{A}\right\|+\varepsilon\|T\|\right) \\
& \leqslant\left\|\left.T\right|_{A}\right\|+\varepsilon_{1}
\end{aligned}
$$

where $\varepsilon_{1}:=\varepsilon(3+2 \varepsilon)\|T\|$. 
Thus $\Gamma\left(\left.T\right|_{M}\right) \leqslant\left\|\left.T\right|_{A}\right\|+\varepsilon_{1}$ and since we can take $\varepsilon_{1}>0$ arbitrarily small, we obtain that $\Delta(T) \leqslant \Gamma(T)$.

Next we present two methods of reducing an arbitrary linear relation to the continuous case.

Definition 15: ([4, IV.3.1]) Given $T \in L R(X, Y)$, let $X_{T}$ denote the vector space $D(T)$ normed by $\|x\|_{T}:=\|x\|+\|T x\|, \quad x \in D(T)$. Let $G_{T} \in L R\left(X_{T}, Y\right)$ be the identity injection of $X_{T}$ into $X$. Then $T G_{T}$ is a bounded linear relation.

Proposition 16. ([4, IV.3.17]) Let $T \in L R(X, Y)$. There exists a normed space $Z$ and a bounded operator $H$ mapping $Y$ onto $Z$ with the following properties:

(i) $H T$ is continuous.

(ii) $H^{\prime}=G_{T^{\prime}}$ and hence $(H T)^{\prime}=T^{\prime} G_{T^{\prime}}$.

(iii) $N(H)=\bar{T}(0)$.

THEOREM 17. For a normed space $X$ the following statements are equivalent:

(i) $X$ is $H I$.

(ii) For every normed space $Y$ and every partially continuous linear relation

$$
T \in L R(X, Y), \quad T \in F_{+}(X, Y) \cup S S(X, Y) .
$$

Proof: (i) $\Rightarrow$ (ii). We observe that if $Z \in I(X)$, then $C(Z)=\{Z \cap N: N \in C(X)\}$ and $P(Z)=\{Z \cap N: N \in P(X)\}$. An application of this observation and the definitions yield immediately the following equivalences:

$$
\begin{aligned}
& T \in P B \Leftrightarrow Q_{T} T \in P B \Leftrightarrow Q_{T} T J_{D(T)} \in P B ; \\
& T \in F_{+} \Leftrightarrow Q_{T} T \in F_{+} \Leftrightarrow Q_{T} T J_{D(T)} \in F_{+} \\
& T \in S S \Leftrightarrow Q_{T} T \in S S \Leftrightarrow Q_{T} T J_{D(T)} \in S S .
\end{aligned}
$$

Hence, we can assume without loss of generality that $T$ is everywhere defined single valued. Let $X$ be a $H I$ and suppose in the first instance that $T$ is continuous. Then $T \notin F_{+} \Leftrightarrow \Gamma(T)=0$ (Proposition 6) $\Leftrightarrow \Delta(T)=0$ (Proposition 14) $\Leftrightarrow T \in S S$ (Proposition 6). For the general case, consider $H T$. Then $H T$ is continuous and from what has been proved for the continuous single valued case, $H T \in F_{+} \cup S S$.

If $H T \in F_{+}$so is $T$ by virtue of $[4, V .2 .16]$. It remains only to prove that $H T$ $\in S S \Rightarrow T \in S S$. To verify this assertion we first show

(4) $T$ partially continuous single valued $\Rightarrow H$ open and $\operatorname{dim} N(H)<\infty \Rightarrow H \in F_{+}$.

Indeed, we note that as $T$ is a partially continuous single valued, $D\left(T^{\prime}\right)$ is a closed finite codimensional subspace of $Y^{\prime}$ and $D\left(T^{\prime}\right)=\left(D\left(T^{\prime}\right)\right)^{\top \perp}[4$, V.11.3]. Consequently, $R\left(H^{\prime}\right)=R\left(G_{T^{\prime}}\right)=D\left(T^{\prime}\right)$ (Proposition 16) $=\left(D\left(T^{\prime}\right)\right)^{\top \perp}=\bar{T}(0)^{\perp}\left(\left[4\right.\right.$, III.1.4]) $=N(H)^{\perp}$ (Proposition 16), so that $R\left(H^{\prime}\right)=N(H)^{\perp}$ equivalently $H$ is open ([4, III.4.6]). Moreover,

$$
\left.N(H)^{\prime}=\left(D\left(T^{\prime}\right)\right)^{\top}\right)^{\prime}=Y^{\prime} /\left(D\left(T^{\prime}\right)\right)^{\top \perp}=Y^{\prime} / D\left(T^{\prime}\right)
$$


and hence $\operatorname{dim} N(H)<\infty$. Thus $H$ is open with finite null space and so by [4, V.5.1], $H \in F_{+}$and (4) holds.

Assume that $H T \in S S$ and $T \notin S S$. Then there exists $M \in I(X)$ for which $\Gamma\left(\left.T\right|_{M}\right)>0$, that is, $\left.T\right|_{M} \in F_{+}$and since $H \in F_{+}$by (4), it follows from [4, V.2.16] that $\left.H T\right|_{M} \in F_{+}$for some $M \in I(X)$ which contradicts $H T \in S S$.

(ii) $\Rightarrow$ (i). Let $M, N \in S(X)$. Since $Q_{M} \notin F_{+}$(as $\operatorname{dim} N\left(Q_{M}\right)=\infty$ ) we have $Q_{M} \in S S$ and hence $Q_{M} J_{N}$ is not injective and open.

The above Theorem fails for linear relations not partially continuous even for operators in Banach spaces. For example, let $T$ be everywhere defined and single valued with $D\left(T^{\prime}\right)=\{0\}$. Then $T \notin P B$ (as $\operatorname{dim} Y^{\prime} / D\left(T^{\prime}\right)=\infty$ ), $T \notin S S$ (since every strictly singular linear relation is partially continuous by [4, V.4.3] and Proposition 6) and as $\beta\left(T^{\prime}\right)=\infty$ is $T^{\prime} \notin F_{-}$and hence $T \notin F_{+}$.

The following result generalises a result of Gowers and Maurey [9] who proved Theorem 18 for bounded operators in Banach spaces. However, their proof is very technical and quite long. Our scheme of proof which can be apply to the particular case of Gowers and Maurey, is very different, it is much shorter and it is based in Proposition 12 and Theorem 17.

TheOREM 18. Let $X$ be a complex $H I$ normed space. Then

$$
P B_{D}(X)=\mathbb{C} I+S S(X) .
$$

Proof: Let $T \in P B_{D}(X)$. By Proposition $12, \sigma_{e+}(T) \neq \emptyset$ and thus there is $\lambda \in \mathbb{C}$ for which $\lambda-T \notin F_{+}$. The result now follows from Theorem 17 .

In general, this Theorem is not true if $T$ is not required to be partially continuous. Indeed, it suffices to note that every strictly singular linear relation is partially continuous by $[4$, V.4.3] and Proposition 6.

\section{REFERENCES}

[1] R.P. Agarwal, M. Meehan and D. O'Regan, Fixed point theory and applications (Cambridge University Press, Cambridge, 2001).

[2] T. Álvarez, R.W. Cross and D. Wilcox, 'Quantities related to upper and lower semiFredholm type linear relations', Bull. Austr. Math. Soc. 66 (2002), 275-289.

[3] T. Álvarez, 'On almost semiFredholm linear relations in normed spaces', Glasgow Math. J. 47 (2005), 187-193.

[4] R.W. Cross, Multivalued linear operators (Marcel Dekker, New York, 1998).

[5] D.E. Edmunds and W.D. Evans, Spectral theory and differential operators (Clarendon, Oxford, Oxford, 1987).

[6] A. Favini and A. Yagi, 'Multivalued linear operators and degenerate evolution equations', Ann. Mat. Pura. Appl. (4) 163 (1993), 353-384.

[7] S. Goldberg, Unbounded linear operators. Theory and applications (McGraw-Hill, New York, 1966). 
[8] L. Gorniewicz, Topological fixed point theory of multivalued mappings (Kluwer, Dordrecht, 1999).

[9] W.T. Gowers and B. Maurey, 'The unconditional basic sequence problem', J. Amer. Math. Soc. 6 (1993), 851-874.

[10] M. Gromov, Partial differential relations (Springer-Verlag, Berlin, 1986).

[11] H.G. Heuser, Functional analysis (Wiley, Chichester, 1982).

[12] M. Muresan, 'On a boundary value problem for quasi-linear differential inclusions of evolution', Collect. Math. 45 (1994), 165-175.

[13] J. Von Neumann, Functional operators II. The Geometry of orthogonal Spaces, Annals of Math. Studies 22 (Princeton University Press, Princeton N.J., 1950).

[14] H. Román-Flores, A. Flores-Franulic, M.A. Rojas-Medar and R.C. Bassanezi, 'Stability of the fixed points set of fuzzy contractions', Appl. Math. Lett. 11 (1998), 33-37.

[15] L. Weiss, 'Perturbation classes of semi-Fredholm operators', Math. Z. 178 (1981), 429-442.

Department of Mathematics

University of Oviedo,

33007, Oviedo

Asturias

Spain

e-mail: seco@uniovi.es 